\title{
LA INSOCIABLE SOCIABILIDAD Y LA TRAMA DE LA FILOSOFÍA KANTIANA DE LA HISTORIA
}

\author{
UNSOCIAL SOCIABILITY AND THE PLOT OF THE KANTIAN PHILOSOPHY OF \\ HISTORY
}

\author{
Eduardo Charpenel* \\ Universidad Panamericana, \\ Ciudad de México D.F. - México. \\ Recibido junio de 2019/Received June, 2019 \\ Aceptado julio de 2019/Accepted July, 2019
}

\begin{abstract}
RESUMEN
En este artículo presento una reconstrucción de la noción kantiana de insociable sociabilidad con el propósito de mostrar su relación con otros elementos claves de la filosofía de Kant como lo son el mal radical, la autonomía moral, y la historia de la humanidad considerada desde un punto de vista heurístico teleológico. Dadas las múltiples conexiones y asociaciones a las que esta noción puede dar lugar, considero que es de suma importancia elucidar cómo entendió Kant nuestras dimensiones de sociabilidad y de insociabilidad, y cómo éstas tienen un impacto en las formas en que desarrollamos nuestras instituciones jurídico-políticas y nuestras prácticas morales. A través del presente análisis pienso que es posible, por un lado, corregir ciertos errores interpretativos como los de Allen W. Wood y Jane Kneller, y, por otro lado, estar en mejores condiciones de apreciar la vigencia del proyecto histórico y antropológico de Kant.
\end{abstract}

Palabras Clave: Kant, sociable insociabilidad, mal radical, filosofía crítica, antropología filosófica, filosofía de la historia.

\begin{abstract}
In this article I present a reconstruction of the Kantian notion of unsocial sociability with the aim of showing its relation with other key elements of Kant's philosophy such as radical evil, moral autonomy, and the history of mankind considered from a heuristic teleological viewpoint. Given the multiple connections and associations this notion can give rise to, I consider of utmost importance to elucidate how Kant understood our social and unsocial dimensions, and how these can have an impact upon the ways we develop our judicial and political institutions and our moral practices. Through the present analysis I believe, on the one hand, that certain interpretative mistakes such as those of Allen W. Wood and Jane Kneller can be corrected, and, on the other hand, that we can be in better conditions to appreciate the ongoing validity of Kant's historical and anthropological project. Key Words: Kant, unsocial sociability, radical evil, critical philosophy, philosophical anthropology, philosophy of history.
\end{abstract}

\section{INTRODUCCIÓN}

La noción de "insociable sociabilidad" (ungesellige Geselligkeit) es peculiar en la trama de la filosofía kantiana. Por un lado, ésta parece ocupar un lugar central en la manera en la que Kant interpreta el desarrollo de la historia humana y en la forma en que concibe la interacción entre los individuos. Alusiones o referencias a esta noción parecen encontrarse en muchos de los desarrollos que Kant presenta en varios textos. Por otro lado, no obstante, el único lugar en donde explícitamente hace mención a dicha noción es en el texto de Idea para una historia universal en sentido cosmopolita ${ }^{1}$

* Autor correspondiente / Corresponding author: echarpen@up.edu.mx 
(Kant, 1784/2006). Por lo mismo, a mi parecer, ha habido un margen de amplitud entre los intérpretes para establecer nexos con otros núcleos teóricos de la filosofía crítica. Sin embargo, considero que no todos los puentes o conexiones que en este sentido se han ensayado son adecuados, sólidos, o sistemáticamente atractivos. En este trabajo, pretendo mostrar cuáles son los problemas que advierto con algunas de las interpretaciones existentes sobre esta noción, entre las que destacan las de Allen W. Wood y Jane Kneller. Como respuesta a las dificultades de dichas propuestas, buscaré desarrollar una lectura en la cual la noción de insociable sociabilidad sea puesta en su justa relación con la noción de mal radical, y sea a su vez interpretada como una clave heurística para leer en términos amplios el despliegue de los talentos y las aptitudes humanas, sin que esto conlleve la disminución de la responsabilidad individual o la sugerencia de que, mediante las instituciones políticas y jurídicas, el progreso moral de los individuos quede ya de suyo garantizado.

Desarrollar una lectura en la que se destaque en qué medida la insociable sociabilidad puede entenderse como un mecanismo que, si bien prepara al ser humano para la moralidad no soslaya como tal la autonomía -e incluso le da ciertos elementos para su efectivo despliegue-, permite sentar bases para una comprensión unitaria de la filosofía kantiana. Así pues, esta revisión de la noción de insociable sociabilidad apunta a establecer un vínculo con las directrices que guían la reflexión kantiana en materia de filosofía de la historia, y también con ciertos rasgos o características que el filósofo de Königsberg tematiza en su concepción antropológica.

Antes de hacer esa revisión crítica puntual de las posturas de los autores antes mencionados, conviene no obstante hacer primero una sucinta reconstrucción de la forma en la que la insociable sociabilidad hace su irrupción en el desarrollo argumentativo kantiano.

\section{LA FILOSOFÍA DE LA HISTORIA KANTIANA Y LA DESTINACIÓN DEL OBRAR HUMANO}

Como ya se hizo mención, el ensayo de la $I a G$ es el marco donde este famoso concepto oxímoron hace su expresa aparición. En el contexto de dicha investigación, Kant pretende realizar una especie de relato en el que se dé cuenta de un propósito que habría de darle sentido narrativo o cierta clase de organización a los esfuerzos humanos, y esto no desde la perspectiva del individuo sino desde la del género. De lo que se trata, pues, es de tratar de establecer un hilo conductor (Leitfaden) que, si bien tentativo, nos ayude a organizar nuestros esfuerzos y acciones como agentes en un marco histórico intergeneracional. Lejos de aspirar a obtener una absoluta certeza sobre la materia -cosa que, conforme a lo que se sugiere, sólo podría alcanzar en un porvenir el Newton que descubra otros mecanismos en el devenir histórico ${ }^{2}-$, Kant da más bien la impresión únicamente de avanzar o poner a consideración un modelo que, en ausencia de uno mejor, sirva como herramienta heurística para la comprensibilidad y articulación de una serie de eventos -eventos que, de no ser puestos en relación de algún modo, posiblemente habrían de generar en nosotros un sentimiento de desasosiego, incertidumbre y escepticismo sobre el sentido del actuar humano en general. Por el carácter particular del conocimiento que Kant busca poner a nuestra disposición, no ha sido raro que los esfuerzos realizados en este y otros escritos de filosofía de la historia sean vinculados con lo que tiempo después, en la Crítica de la facultad de juzgar, se habrá de tematizar detalladamente bajo el juicio teleológico reflexionante ${ }^{3}$.

$Y$ en efecto, en el marco de $I a G$ se parte desde un primer momento de premisas teleológicas cuando se afirma en la primera proposición que "todas las disposiciones naturales de una criatura están determinadas a desarrollarse alguna vez de manera completa y adecuada" (IaG, Vol. AA VIII, p. 18 /Kant, 2006, p. 75). Este aserto, si bien evoca inmediatamente formulaciones aristotélicas según las cuales la naturaleza no hace nada en vano ${ }^{4}$, no está expresado, a diferencia del Estagirita, desde la perspectiva constitutiva del andamiaje básico y fundamental de la realidad, sino de consideraciones regulativas bajo el arreglo de nuestra facultad de juzgar. Así pues, las afirmaciones realizadas en el escrito acerca de la destinación del género humano no son simples generalizaciones. Éstas son más bien reflexiones que hilvanan un material inicialmente disperso de nuestro conocimiento del mundo, dándole al mismo un orden teleológico-racional que responde a la estructura de nuestras facultades cognitivas. Este camino conduce a Kant a afirmar de modo crucial en la segunda y tercera proposición de $I a G$ que las disposiciones naturales deben de 
desarrollarse por completo en la especie y que la felicidad o realización a la que hemos de aspirar no hemos de obtenerla sobre la base del instinto sino de lo que fragüemos a partir de la razón.

Es posible apreciar una conexión entre estas dos tesis kantianas. Por un lado, tenemos el dato de experiencia de la finitud humana, la cual implica, entre otras cosas, que los seres humanos tenemos necesidad de aprender unos de otros a través de las generaciones, pues de lo contrario cada individuo estaría condenado a obtener por sus propios medios, de nueva cuenta, una y otra vez, el conocimiento de todas las disciplinas y saberes con innumerables equívocos y tropiezos -únicamente para que, al final del día, esta labor quede fundamentalmente inconclusa y sin haber dejado fruto alguno. Por otra parte, podemos razonablemente conjeturar que la naturaleza busca que obtengamos una realización por nuestros propios esfuerzos, y esto no podría darse mediante el simple dictado de los instintos ${ }^{5}$. Más aún, incluso podría decirse que la condición para la realización humana a partir de la libertad -y esto, como se verá más adelante, tendrá importantes repercusiones en la idea misma de sociabilidad-es precisamente esta precariedad en los talentos y disposiciones que posee el ser humano, el cual, en comparación con el animal, parece estar esencialmente desvalido o desprovisto cuando llega al mundo. Por decirlo de otra manera, parecería que el ser humano tiene, por esta inmediata menesterosidad suya, las cosas mucho menos resueltas que el resto de los seres vivos.

Con estos desarrollos anteriores como sostén, en la cuarta proposición de $I a G$ se introduce la noción de antagonismo (Antagonismus) para dar cuenta, de un modo más puntual, sobre cómo la naturaleza ha planeado o dispuesto ese despliegue para la adquisición de los talentos por parte del ser humano. Este antagonismo se vincula a su vez con la idea misma de insociable sociabilidad. Por la importancia del pasaje de cara a nuestro propósito conviene traerlo explícitamente a consideración:

Entiendo aquí por antagonismo la insociable sociabilidad del hombre; es decir, la misma inclinación a caminar hacia la sociedad está vinculada con una resistencia opuesta, que amenaza continuamente con romper esta sociedad. Esta disposición reside ostensiblemente en la naturaleza humana. El hombre posee una propensión a entrar en sociedad, porque en tal estado se siente más como hombre, es decir, siente el desarrollo de sus disposiciones naturales. Pero también tiene una inclinación mayor a individualizarse (aislarse), pues encuentra igualmente en sí mismo la cualidad insociable, que le lleva a conducir todo meramente conforme a lo que su sentido disponga y a esperar, por ello, resistencia por todas partes, del mismo modo que sabe que, por la suya, es propenso a la resistencia contra los demás. (IaG, Vol. AA VIII, pp. 21-22 / Kant, 2006, p. 78).

Estas disposiciones antagónicas en el ser humano -cuyo tratamiento análogo se puede apreciar, con diferentes enfoques y matices, en otros autores de la modernidad temprana como Grocio, Hobbes, Hutcheson y, por supuesto, Rousseau ${ }^{6}$ son tomadas por Kant como tendencias que tienen cada una sus respectivos dividendos. Así pues, por un lado, la sociabilidad le permite al ser humano, a través de la convivencia con otros, reconocerse genéricamente -es decir, establecer lo que es propio o característico de aquellos seres como él-, desarrollar toda una serie de aptitudes, y gozar de varios bienes que de otra forma no estarían disponibles para él. Por otro lado, observamos sin embargo que ese movimiento es seguido de un rechazo o una confrontación, toda vez que busca actuar sólo conforme a su idiosincrasia, impulso o convicción, lo cual quiere decir que, en ese contexto comunitario, busca o intenta hacer las cosas a su modo, topándose con una resistencia (Widerstand) que nuevamente lo lleva a apartarse ${ }^{7}$. Sin embargo, aquella resistencia, como se aclara en lo sucesivo,

le lleva a superar su inclinación a la pereza
y, movido por el ansia de honor, de poder o
de bienes, a procurarse un rango entre sus
congéneres, a los que no puede soportar,
pero de los que tampoco puede prescindir.
(IaG, Vol. AA VIII, p. 22 / Kant, 2006, p. 78).

Sobre estas bases, la cultura misma se va desarrollando, se va formando el gusto, y en un proceso de progresiva Ilustración, nos dice Kant, los esfuerzos humanos dan lugar a "una manera de pensar, que puede transformar, con el tiempo, la ruda disposición natural para la discriminación ética en principios prácticos determinados $\mathrm{y}$, por fin, de este modo, una concordancia en sociedad, 
patológicamente provocada, en un todo moral" (IaG, Vol. AA VIII, p. 22 / Kant, 2006, p. 79). Este último punto no es en definitiva menor, pues Kant parece dar a entender precisamente que de esta dinámica de concordia y enemistad es posible esperar un desenlace de cierta índole moral, lo cual, a todas luces, podría parecer algo insólito o improcedente puesto que, en conformidad con el resto de los escritos de su obra, tendemos a asociar lo propiamente moral con aquello que dimana del respeto por la ley moral misma y del ejercicio de la autonomía -categorías respecto a las cuales no parece haber punto intermedio alguno.

Dejemos por lo pronto ese último punto en suspenso. Más adelante volveremos al mismo con los elementos que recojamos a partir de la discusión. Lo que cabe decir ahora es que en las siguientes proposiciones de $I a G$-mismas que, por una cuestión de espacio, es imposible reconstruir aquí- se habla de tareas que vendrían aparejadas con lo anterior, a saber, la constitución de una sociedad civil, el establecimiento de un estado de derecho, el mejoramiento de los gobernantes, y la relación pacífica y cosmopolita entre los pueblos, para finalmente llegar a un cierre en el que se hacen ciertas consideraciones de corte arquitectónico sobre la importancia y alcances de una propuesta de esta índole. Todos estos núcleos teóricos, como se puede apreciar, son tópicos a los que Kant recurrentemente vuelve en sus escritos sobre filosofía de la historia y filosofía jurídica, pero en el contexto específico de $I a G$ no cabe duda de que aquello que parece darle cohesión al relato presentado -mismo que el propio Kant llega incluso a equiparar, en la última proposición del escrito, con una novela (Roman), pero una que sería de importancia mayúscula (IaG, Vol. AA VIII, p. 29 / Kant, 2006, p. 89)- es la insociable sociabilidad. En pocas palabras, siguiendo la ilustrativa metáfora empleada por Höffe, podríamos incluso considerar a la insociable sociabilidad como el "motor" de la historia (Höffe, 2011, p. 14). Por ende, pareciera ser que una comprensión lograda de este proceso no puede renunciar a entender en sus detalles más propios los elementos que la componen. $Y$ en este sentido, gran parte de descifrar lo que significa la insociable sociabilidad pasa por entender qué significa para Kant lo insociable y lo sociable. En vistas a que la caracterización aristotélica del hombre como un animal político ha permeado tanto el imaginario filosófico de occidente, se vuelve incluso todavía más importante elucidar lo que implica la insociabilidad en este marco. Dos intérpretes que en este sentido han brindado sugerentes propuestas son Allen W. Wood y Jane Kneller. Debido al alcance de estas lecturas, considero relevante someter sus propuestas a una discusión crítica, puesto que aprecio que ellos vinculan con la noción de insociable sociabilidad ciertos elementos de la filosofía kantiana con los que no se encuentra en plena sintonía -o, al menos, hacen estos vínculos con ciertos énfasis que considero inadecuados.

\section{DOS LECTURAS SOBRE LA INSOCIABLE SOCIABILIDAD}

Comenzaré primero con la lectura de Allen W. Wood. Este intérprete ha desarrollado, ya desde hace tiempo, una lectura en la cual lo que se busca enfatizar es la estrechísima vinculación -incluso, me atrevería a afirmar, homogeneidad-que existe, a su juicio, entre la insociable sociabilidad y la noción de mal radical (radikales Böse) que Kant aborda en el marco del escrito sobre la Religión. En el coloquialmente llamado Religionsschrift, Kant busca desarrollar una lectura en la cual los contenidos básicos de una religión racional queden delineados explicitados, y la forma de hacer esto consiste en examinar los preceptos y las doctrinas que caracterizan a la religión que, en consideración del filósofo de Königsberg, en términos fácticos e históricos, es la más próxima a lo que la razón práctica prescribe, a saber, la religión cristiana. En este sentido, la noción de pecado original es revisitada para hacerla inteligible, pues no cabe duda alguna de que prima facie resulta chocante para la razón la idea de que uno sea culpable o deudor de algo que uno mismo no cometió sino los más remotos antepasados. De esta forma, las Escrituras se convierten en el análisis kantiano en un fértil material que abona para una reflexión donde, a través de la guía que marca la categoricidad de la ley moral, ciertos contenidos son depurados y otros -tomados por irrelevantes o míticos- son rechazados. En el curso de su argumentación sobre esta materia, como es sabido, Kant llega a la conclusión de que el mal radical en el hombre hay que remontarlo a la Gesinnung o disposición fundamental del ánimo, misma que es la máxima básica de nuestro carácter moral.

El complejo análisis kantiano sobre el mal pasa por dos momentos; un primer momento que 
es de corte un tanto más individual y centrado en la estructura de la subjetividad humana, y otro que es de corte netamente colectivo. Los dos son aprovechados por Wood para subrayar el fundamento antropológico del mal radical, el cual no es otro que la insociable sociabilidad ${ }^{8}$. Generalmente, la estrategia argumentativa de Wood ha pasado por indicar que el mal radical en Kant, en último término, sólo es comprensible si es que éste se aclara por nuestra experiencia de la insociable sociabilidad. Algunos intentos ya se han ensayado para mostrar que Wood no hace justicia a la noción de mal radical precisamente por buscar entenderla desde la insociable sociabilidad ${ }^{9}$. Pero a mi parecer esto vale también para el otro lado de la moneda: la insociable sociabilidad queda también desdibujada mediante esta equiparación.

Como se mencionó, Wood intenta subrayar la equivalencia o los vasos comunicantes entre una y otra noción a partir de dos momentos fundamentales. Así pues, en un primer momento, este autor hace hincapié en que una de las disposiciones de los individuos para el bien (Anlagen zum Guten), a saber, la disposición para la humanidad (Menschheit), puede constantemente devenir en algo propiamente inmoral. Si bien esta disposición está orientada de suyo a un amor propio ordenado y a un sentido de igualdad de los individuos frente a los otros seres humanos, la misma es susceptible de ser contaminada por consideraciones que promueven una férrea competitividad entre los mismos. Sobre la disposición todavía moral de no buscar que nadie busque arrogarse injustamente una superioridad sobre nosotros "pueden injertarse los mayores vicios de hostilidades secretas o abiertas contra todos los que consideramos como extraños para nosotros" ( $R G V$, Vol. AA VI, p. 16 / Kant, 2001, p. 44) ${ }^{10}$. De acuerdo con Kant, estos vicios provienen no de la naturaleza misma sino de la cultura (Kultur), y cuando éstos alcanzan su epítome o máximo tienen la forma de la envidia, la ingratitud y la alegría del mal ajeno y son llamados "vicios diabólicos" (teuflische Laster) (RGV, Vol. AA VI, p. 17 / Kant, 2001, p. 45).

Vemos pues que, en una línea semejante a la trazada por Rousseau, Kant identifica toda una serie de patologías en sentido moral que tendrían propiamente hablando su sede en la cultura y en la convivencia humanas -como si, por decirlo de algún modo, la convivencia de los seres humanos estuviese ya viciada desde un primer momento.
Wood se sirve de ello para subrayar la idea de que, en realidad, cuando pensamos en el mal, esta clase de prácticas son nuestro primer analogado o principal referente.

Esto nos lleva a un segundo momento destacado por Wood, en el cual Kant, después de haber hecho un análisis de la subjetividad y del carácter inteligible del individuo, traslada en los libros tercero y cuarto del Religionsschrift el enfoque directamente sobre el colectivo humano. Y es ahí donde encontramos un pasaje que parece abonar de un modo muy enfático a la propuesta desarrollada por Wood:

No es por los estímulos de la primera [i.e., de la naturaleza; agregado propio] por lo que se despiertan en él las pasiones, que así propiamente han de ser llamadas, las cuales ocasionan tan grandes estragos en su disposición originalmente buena. Sus necesidades son sólo pequeñas y su estado de ánimo en el cuidado de ellas es moderado y tranquilo. Sólo es pobre (o se tiene por tal) en la medida en que recela de que otros hombres le tienen por tal y podrían despreciarle por ello. La envidia, el ansia de dominio, la codicia y las inclinaciones hostiles ligadas a todo ello asaltan su naturaleza, en sí modesta, tan pronto como está entre hombres, y ni siquiera es necesario suponer ya que éstos están hundidos en el mal y constituyen ejemplos que inducen a él; es bastante que estén ahí, que lo rodeen, y que sean hombres, para que mutuamente se corrompan en su disposición moral y se hagan malos unos a otros. (RGV, Vol. AA VI 93-94 / Kant, 2001, pp. 117-118).

El hecho de que en este pasaje se cifre desde un primer momento la convivencia humana en estos términos de recelo, competitividad y violencia sugiere que, en efecto, Kant es un enorme deudor de Rousseau, y parece darle un fundamento antropológico y social al mal de mucho mayor peso que a uno de cualquier otra índole ${ }^{11}$. Así pues se podría pensar que, en último término, cualquier tipo de análisis en clave trascendental -como el que parece realizar Kant sobre todo en los dos primeros libros del Religionsschrift-palidece ante esta dimensión cultural y comunitaria del mal que hace irrupción tan pronto buscamos establecer un trato con nuestros congéneres. Por lo mismo, la solución del mal ha de ser también una propiamente social: el establecimiento de una comunidad ética 
(ethisches Gemeinwesen) o una iglesia invisible (unsichtbare Kirche).

Existen problemas a mi entender, tanto de orden exegético como de orden sistemático, con la lectura de Wood. Pero considero más apropiado tratarlos, en conjunto con las objeciones que habré de presentar ante la lectura de Jane Kneller, en el marco del siguiente apartado. Pienso que, de esta forma, los contornos de la interpretación que aquí se busca defender quedarán más claros. Esto, a su vez, nos dará ocasión de conectar ciertas premisas de nuestro análisis con algunas consideraciones más amplias sobre la naturaleza de la historia humana en la filosofía kantiana.

Pasemos, pues, a la revisión de la propuesta de Kneller. De acuerdo con esta autora, el principio de la insociable sociabilidad se encuentra arropado en $I a G$ por la concepción kantiana de la Ilustración (Kneller, 2011, p. 46). Kant ve necesario que el hombre se cultive, se perfeccione y salga de la rudeza con la que llega al mundo y en la que se mueve, de una forma ya más sofisticada, en su interacción con los otros en las sociedades modernas. En términos generales, la visión de la humanidad que se nos presenta en $I a G$ es -a pesar de lo que pudieran sugerir ciertos logros civilizatorios- una más bien de corte hobbesiano (Kneller, 2011, p. 53). Por ende, enfatiza la autora, existe una exhortación a abandonar nuestra libertad salvaje, la cual es equiparada a la de los árboles que crecen al despoblado esparciendo sus ramas por toda la tierra (IaG, Vol. AA VIII, p. 22 / Kant, 2006, p. 80). Al privilegiar la armonía que es fruto de la recíproca limitación que se da entre los árboles que crecen unos junto a otros, Kant expresa su convicción, incluso de orden estético, de que el orden y la simetría son mejores que las formas libres y caóticas (Kneller, 2011, p. 53). De ahí pues que Kant tenga en este escrito bases más bien pesimistas que fomenten la disciplina y no idílicas que privilegien la espontaneidad y el libre e ininterrumpido desarrollo de la personalidad de los individuos.

La propuesta de Kant es una que apunta hacia un orden mundial cosmopolita. Esto podría parecer quimérico o utópico y, a juicio de Kneller, el filósofo de Königsberg es perfectamente consciente de ello y busca escapar por todos los medios que a su teoría se le acuse de ingenuidad o de inocencia. Los más horribles hechos políticos de la historia humana tienen que encontrar cabida de algún modo en un relato filosófico que apueste por un orden político mundial de la índole aquí planteada. Es decir, el proyecto kantiano es uno que, en la lectura de Kneller, trata de comprender "todo el mal social como pasos necesarios hacia un futuro" (Kneller, 2011, p. 54). Por ello, se busca dar cabida en el mismo al mal para darle a su propuesta una credibilidad realista -una propuesta que ha de tomar en consideración los acontecimientos históricos violentos y crueles como parte de su propia narrativa.

Lo anterior no significa, sin embargo, que el orden cosmopolita tenga realmente como eslabón o elemento necesario la insociabilidad del hombre. De acuerdo con el razonamiento de esta intérprete, si Kant apunta al abandono de nuestra libertad salvaje es porque "debe haber otra forma que la del egoísmo y la de la insociabilidad en la cual los seres humanos también vivamos la libertad" (Kneller, 2011, p. 54). Y ése es el componente propio de la sociabilidad. Por ello, Kneller subraya que el proyecto cosmopolita concebido por Kant en $I a G$ y desarrollado ulteriormente en otros escritos debe descansar fundamentalmente sobre este elemento y considerar al otro, desde otro punto de vista, hasta cierto punto como accidental o prescindible. Así pues, Kneller opta más bien por hablar de un proceso de sociable sociabilidad (gesellige Geselligkeit) como lo más emblemático del pensamiento kantiano (Kneller, 2011, p. 54).

Esta sociabilidad la encuentra Kneller ejemplificada en ciertos tratamientos de lo bello en la $K U$. Un pasaje ilustrativo a este respecto sería el siguiente:

Desde un punto de vista empírico, lo bello sólo interesa en la sociedad. Y si se concede que el impulso social es el impulso natural del hombre, más la idoneidad y la tendencia a ella, esto es, la sociabilidad, se concede como propiedad que pertenece a la humanidad, no puede faltar entonces que también deba considerarse al gusto como una capacidad de enjuiciamiento de todo aquello por medio de lo cual uno puede comunicar su sentimiento a todos los demás, o sea, como medio de promoción de aquello que reclama la inclinación natural de cada uno. ( $K U, \mathrm{Vol}$. AA V, pp. 296-297 / Kant, 2003, p. 262).

En el goce propio que encontramos de ciertos placeres o prácticas en la compañía de 
otros seres humanos advertimos de modo claro nuestra vocación a vivir en comunidad. Aquí la humanidad se encuentra retratada en términos de convivencia armónica y de comunicabilidad de ciertas experiencias que marcan profundamente el ánimo. Kneller destaca en este sentido las curiosas palabras de Kant según las cuales este impulso por lo bello en el contexto social es equiparable a un "contrato social original (ursprünglichen Gesellschaftsvertrag) que es dictado por la humanidad" (KU, Vol. AA V, p. 296 / Kant, 2003, p. 262). Esta formulación parece sugerir precisamente que, todo lo que sea contrario o adverso a los intereses que fomenten esta humanidad, habría que tomarlos como cuestiones o aspectos que no son esenciales a la misma. Por este motivo, es de pensarse que los impulsos o inclinaciones que contravengan a estas experiencias comunicativas habría más bien que considerarlos como elementos no esenciales de la identidad del tipo de seres que somos.

El interés empírico por lo bello natural encuentra una contraparte de corte moral-práctico, según subraya Kneller, en la preocupación humana de que los fines de la moralidad prevalezcan en los distintos ámbitos de nuestra convivencia -esta contraparte positiva difícilmente se habría de encontrar, conforme a lo que ella argumenta, en la dinámica de la insociable sociabilidad. La sociable sociabilidad que destaca esta comentadora parecería ser un proceso que, una vez iniciado, tiene posibilidades de éxito mayores que el que se encuentra presente en $I a G$. De modo que, prácticamente a modo de exhortación, Kneller sugiere más bien "concentrarse en nuestra estima por comunicar formas bellas naturales y reproducir las mismas" en el ámbito de nuestra conducta (Kneller, 2011, p. 53). Aquella disciplina y orden que en $I a G$ parecían ser estrictamente exigidas de cara al establecimiento del orden cosmopolita podrían darse sobre otras bases, a saber, las que nos brindan la imaginación y el gusto, como nos lo sugiere el siguiente pasaje:

Por así decirlo, el gusto hace posible el tránsito del estímulo de los sentidos al interés moral habitual, sin que sea un salto excesivamente violento, en la medida en que también representa a la imaginación en su libertad como teleológicamente determinable para el entendimiento, y enseña a encontrar una satisfacción libre incluso en objetos de los sentidos, también sin el estímulo de los sentidos. ( $K U$, Vol. AA V, p. 354 / Kant, 2003, pp. 328-329).

Según nos dice esta intérprete, la imaginación "necesita ejercicio para corresponder al entendimiento y para poderse desarrollar ulteriormente" (Kneller, 2011, p. 58). Esto se vería reflejado en la manera en cómo el genio artístico -y posiblemente el espectador u observador también- tienen que renunciar a la extravagancia y dar una forma, ya sea en la creación o en la contemplación, a aquello que tienen ante sí. Y esto, potencialmente, con arreglo a nuestra propia humanidad, parecería al menos ser replicable o esperable en el plano de nuestra interacción jurídica, política y social.

\section{LIBERTAD, HISTORICIDAD Y PROGRESO}

Las lecturas anteriormente revisadas son sugerentes porque destacan elementos con los que una interpretación satisfactoria de la noción de insociable sociabilidad debe de lidiar. En cierto sentido, me atrevería incluso a decir que estas lecturas tienen un carácter diametralmente opuesto entre sí: por una parte, la de Wood subraya un fundamento antropológico del mal y de la insociabilidad, con lo cual pareciera ser que el ser humano se encuentra, en el ámbito de su convivencia, ya radicalmente marcado, en términos negativos, por las propensiones características de su naturaleza, y que sólo mediante un gran esfuerzo de carácter social podrá él revertir esta tendencia; por otra parte, observamos que Kneller relativiza la dinámica de la insociable sociabilidad presentada en $I a G$ al poner de relieve los intereses empíricos como la comunicabilidad de nuestras experiencias estéticas que serían, a juicio de ella, lo más propio y destacado de la humanidad, con lo cual lo que quedaría en un primer plano sería, tal como ella la llama, la dinámica de la "sociable sociabilidad".

Como hacía mención, aunque reconozco una importante valía en los aportes de estos autores, considero que las lecturas ensayadas por ambos yerran en varios puntos decisivos. Quisiera exponer ahora en términos generales lo que considero son notas fundamentales para comprender adecuadamente la noción de insociable sociabilidad -en el marco de esta discusión, se harán manifiestos mis desacuerdos con estos dos intérpretes. Para estos efectos, me apoyaré en algunos de los puntos 
que se expusieron en la reconstrucción del pasaje kantiano de la cuarta proposición de $I a G$ en la segunda sección del presente artículo, pero siendo éstos cobijados o elucidados a través de la noción de mal radical, la cual considero como clave en el entramado crítico kantiano.

A mi juicio, una interpretación sobre el mal en la filosofía kantiana -incluso si lo que se busca poner en un primer plano es la dimensión social del mismo- no puede soslayar el desarrollo argumentativo de los primeros libros del Religionsschrift ${ }^{12}$. Por supuesto, aquí es imposible ofrecer una reconstrucción puntual del análisis que el filósofo ofrece sobre esta materia ${ }^{13}$. Lo que se puede decir a grandes rasgos sobre el mismo es que éste apunta a que el mal tiene en el ser humano, en último término, un origen inescrutable e infechable en el marco de la temporalidad. El mal no se puede adjudicar en Kant a causas externas, porque de lo contrario tendríamos que suponer un origen no moral de algo moral. Por consiguiente, hemos de pensar que el mal es una especie de elección fundamental realizada por nosotros, fuera de la temporalidad, en nuestra dimensión inteligible o nouménica, pero que tiene una repercusión sobre nuestra naturaleza empírica o fenoménica -en la cual ciertamente el mal encuentra diversas formas de manifestación culturales y sociales que, en otras circunstancias u otros momentos de convivencia, no podrían hacerse patentes ${ }^{14}$. El mal radical para Kant tiene un componente de irracionalidad e inexplicabilidad en tanto que no podemos dar cuenta de por qué se incurre en éste, toda vez que, de haber un motivo determinante, estaríamos condicionados, y, por lo tanto, exculpados de toda posible responsabilidad por el mismo. No obstante, sin embargo, si éste ha de sernos imputado, entonces tenemos que pensarlo como habiéndolo escogido libremente, aunque el motivo detrás de esto, por el propio carácter de inescrutable del mal, no sea susceptible de ser identificado. Por ello, el análisis del mal tiene que partir de un análisis trascendental de lo que es propio de nuestro carácter inteligible -análisis que, por supuesto, no pierde nunca de vista la condición empírica antropológica, pero que en definitiva no descansa sólo en esta última-, el cual es susceptible de ser entendido mediante un sistema de un conjunto de máximas de mayor y menor jerarquía, respecto a las cuales la Gesinnung sería aquella más fundamental y de mayor peso. Si esto es así, entonces otras formas o expresiones del mal, como hacía mención, tienen que tener esto como su fundamento ${ }^{15}$.

Además de ser esta una lectura que hace mayor justicia a los demás textos de la obra kantiana, considero que es a su vez sistemáticamente más atractiva, toda vez que otras opciones corren el riesgo, como recién apuntaba, de diluir lo propiamente característico del mal como fenómeno moral, a saber, ser un fenómeno de la libertad. Si lo que uno sugiere es que el mal proviene o es inherente a nuestra propia condición antropológica social, lo que a mi entender se desprende de ello es que no termina por quedar en claro a quién habría que atribuírselo ${ }^{16}$. En todo caso, sin embargo, no serían los individuos concretos a quienes habría que adjudicárselo, y por lo mismo no podríamos responsabilizarlos por su conducta.

Ahora bien, por otra parte, es cierto como apuntábamos antes que el mal puede tener manifestaciones de insociabilidad ${ }^{17}$, y más aún, puede generar mecanismos de convivencia en los que los individuos sean particularmente proclives de incurrir. Estos mecanismos o sistemas de convivencia son en los que, a mi parecer, Kant busca tematizar en el marco del escrito de $I a G$, y esto no con el fin de subrayar con un carácter de necesidad absoluta el que tales o cuales eventos históricos fueron inevitables -como parecía sugerir Kneller al hablar, en su análisis de $I a G$, de todo mal social como necesario para un futuro mejor (Kneller, 2011, p. 53)- sino simplemente para indicar que el mal, desde una perspectiva teleológica reflexionante, no es arbitrario -que no es lo mismo que decir que es necesario ${ }^{18}-$, y más aún, que es posible esperar que del mismo se obtenga algún beneficio, como de hecho ocurre con la creación de la sociedad civil y de las instituciones que, frente a las tendencias insociables del hombre, buscan normar la convivencia humana. Existe ciertamente, conforme a la tercera proposición de $I a G$, un plan de la naturaleza, pero éste consiste precisamente, como ya hemos visto, en que los seres humanos logren su felicidad y realización a partir de sus propios esfuerzos, es decir, a partir del ejercicio de su libertad. De lo que se desprende también de ese plan de la naturaleza es que los efectos de los malos o inadecuados usos de la libertad puedan ellos mismos ser aprovechados en un segundo momento. Pero Kant no sugiere que este proceso sea lineal o que no pueda haber retrocesos en el mismo. A este respecto -en contra 
de lo que sugiere Kneller- es posible mencionar dos casos de este carácter "abierto" de la historia en clave kantiana. En primer lugar, al analizar en $S F$ la naturaleza de la Revolución Francesa como un hito en la historia humana que despierta el entusiasmo de todos quienes lo contemplan, Kant no duda en afirmar que ese movimiento social no tiene ningún éxito garantizado y que bien puede fracasar ( $S F$, Vol. AA VII, p. 88 / Kant, 2003, p. 164). En segundo lugar, una aparente inconsistencia en $I a G$ es que, por un lado, Kant afirma en la sexta proposición que, para que el orden cosmopolita se dé, los gobernantes deben ellos mismos mejorar moralmente, pero, por otro lado, también se dice en la séptima proposición que para estos efectos es necesario que ya existan marcos institucionales adecuados. Considero, siguiendo a Otfried Höffe, que en realidad Kant, al hacer estas aseveraciones, no está incurriendo en una contradicción: no es que el filósofo esté tratando de decir que una cuestión sea condición de la otra, sino que ambas deben de lograrse ${ }^{19}$. En efecto, nadie puede prever si un gobernante, con un buen talante moral, habrá de lograr mediante sus reformas que los ciudadanos se vean movidos -que no determinados- a ser mejores personas, o bien que un pueblo, en virtud de sus cualidades, no genere cambios que en un momento dado tengan una repercusión en los mismos gobernantes y las instituciones. Estos dos factores hablan, a mi juicio, a favor de entender el desarrollo de la historia humana como un proceso que, si bien puede pensarse o concebirse como si estuviese dirigido $\mathrm{u}$ orientado a un fin -que no sería otro que la consolidación de gobiernos cosmopolitas y una moralización del género humano-, se encuentra en los acontecimientos particulares esencialmente abierto.

Por lo demás, vale la pena comentar -a fin de aclarar el calado de la filosofía kantiana de la historia- que lo dicho anteriormente ha sido algunas veces cuestionado. El caso paradigmático al respecto es el de Hannah Arendt. En palabras de esta autora:

El progreso infinito es la ley de la especie humana; al mismo tiempo, la dignidad del hombre exige que él sea considerado (en cada uno de nosotros) en su particularidad, y, como tal, que sea visto - pero sin comparación alguna $\mathrm{y}$ de forma independiente al tiempo- como si reflejara a la humanidad en general. En otras palabras, la misma idea de progreso - si éste es más que un cambio de circunstancias y un mejoramiento del mundo- contradice la noción de Kant de dignidad humana. Es contrario a la dignidad creer en el progreso. (Arendt, 1989, p. 77).

Arendt parte de la idea de que los seres humanos, al participar en este proceso descrito por Kant, estarían de algún modo siendo manipulados. Si ellos coadyuvan con sus acciones a un fin que ellos mismos no han consentido, pareciera ser que sólo son instrumentos de algo que ocurre por detrás de sus espaldas. Pero en realidad, como se ha venido apuntando, esto no ha de entenderse como un proceso necesariamente lineal y uniforme hacia lo mejor, como Arendt parece tener aquí en mente. Para Kant son los individuos, con base en sus inclinaciones, apetitos y preferencias naturales que, al decidir seguirlas o no, van generando ellos mismos estos cambios en la sociedad, y van influyendo, aunque ellos no se percaten de ello, en la manera en que otros individuos en lo sucesivo habrán de actuar, sin que esto tenga en ningún sentido un carácter plenamente determinante. Con su objeción, Arendt parece sugerir que los seres humanos somos capaces de tener un control absoluto tanto de la forma en que nos posicionamos frente al contexto donde nos tocó nacer, como de la manera en que hemos de buscar desarrollarnos de aquí en lo sucesivo, sin que hubiera ninguna clase de caminos ya allanados previamente que marquen una pauta para nuestras acciones. Al ser estas condiciones irrealizables e indisponibles, no me parece que la dignidad humana pueda ser violentada, como Arendt lo expresa, por la misma idea de progreso $^{20}$.

Pero volviendo a nuestro tema principal: es ingenuo pensar, como sugiere Kneller, que el proceso de la insociable sociabilidad pueda, simplemente, ser sustituido por aquel de la sociable sociabilidad del que ella habla ${ }^{21}$. Esto no parece ser una cuestión simplemente de decidir optar o tomar un camino u otro, y, en cualquier caso, no se trata de procesos que un solo individuo pueda decidir poner en marcha a partir de su mera voluntad, sino que lo que está en juego, como hemos podido apreciar, tiene que ver con ciertos patrones y órdenes que se van dando desde la esfera de la acción colectiva.

En la misma línea, podemos decir que también es ingenuo pensar que este proceso 
generará sin más la perfección o santidad morales del género humano, sobre todo si, como hacíamos mención en la introducción al presente trabajo, la autonomía hubiera de ser pensada bajo un esquema de blanco y negro. Pienso que esto no tiene por qué ser el caso. Apelando a la idea de cultura de la disciplina (Cultur der Zucht) kantiana, podemos representarnos este proceso de la insociable sociabilidad como uno en el cual, si bien no nos volvemos necesariamente mejores, al menos vamos adquiriendo progresivamente un dominio sobre nuestros propios apetitos e impulsos, de modo que para los seres humanos que recorren este proceso es más favorable el ejercicio de su agencia moral ${ }^{22}$. Esto se vincula a mi entender de modo muy natural con la idea kantiana según la cual, si bien nosotros nunca podemos estar absolutamente ciertos de que somos individuos buenos - pues pensar tal cosa sería un síntoma inequívoco de fariseísmo-, sí podemos tener cierta certeza sensible de que vamos haciendo cierto progreso en nuestro actuar en la medida en que, paulatinamente, vamos adquiriendo cierta fortaleza moral ${ }^{23}$. Pienso pues que, si la dinámica de la insociable sociabilidad sienta esas bases para una posible mejora del género humano, no es poca cosa lo que con ella se logra.

\section{CONCLUSIÓN}

La dinámica de la insociable sociabilidad representa un núcleo teórico en el cual se vertebran muchas de las inquietudes históricas, morales, políticas, jurídicas e incluso hasta teológicas del pensamiento kantiano. Debido a los vasos comunicantes a los que da margen, no resulta sorprendente que se hayan ensayado interpretaciones, como las aquí examinadas, en las que otros elementos de la filosofía crítica poco considerados parecían irrumpir para jugar un papel determinante. Considero, no obstante, que las claves que he buscado brindar aquí respecto a la debida ubicación arquitectónica de la insociable sociabilidad frente a otros núcleos teóricos de enorme importancia como la proyección teleológica de la historia, el mal radical y la autonomía moral -por sólo mencionar algunospermiten, por un lado, hacer frente a distintas objeciones que se le suelen hacer a la propuesta kantiana, y por otro lado, y más importante, pueden ser de utilidad para apreciar en su justa medida el valor perenne de estos instrumentos conceptuales kantianos, que todavía fungen como claves heurísticas muy favorables para releer la historia previa del género humano, comprender nuestro presente, e imaginar-que no profetizar- hacia dónde es deseable que nuestras diversas instituciones y prácticas progresen en un futuro. 


\section{Referencias}

Aristóteles. (1978). Acerca del alma. Madrid: Gredos.

Aristóteles. (2000). Partes de los animales. Marcha de los animales. Movimiento de los animales. Madrid: Gredos.

Allison, H. (2001). Ethics, Evil, and Anthropology in Kant: Remarks on Allen Wood's Kant's Ethical Thought. Ethics, 111(3), 594-616.

Anderson-Gold, S. (2001). Unnecessary Evil. History and Moral Progress in the Philosophy of Immanuel Kant. Albany: SUNY.

Arendt, H. (1989). Lectures on Kant's Political Philosophy. Chicago: Chicago University Press.

Arendt, H. (1998). The Human Condition. Chicago: Chicago University Press.

Brandt, R. (2010). Immanuel Kant - Was bleibt? Hamburgo: Felix Meiner.

De Haro Romo, V. (2015). Duty, Virtue and Practical Reason in Kant's Metaphysics of Morals. Hildesheim: Olms.

Höffe, O. (2006). Kant's Cosmopolitan Theory of Law and Practice. Cambridge: Cambridge University Press.

Höffe, O. (2011). Einführung. En O. Höffe (Ed.), Immanuel Kant. Schriften zur Geschichtsphilosophie (pp. 1-28). Berlín: Akademie Verlag.

Kant, I. (1910ss.). Kant's gesammelte Schriften, Königlich preussischen Akademie der Wissenschaften. Berlín: Walter de Gruyter.

Kant, I. (2001). La Religión dentro de los límites de la mera razón. Madrid: Alianza.

Kant, I. (2003). Crítica del discernimiento. Madrid: Mínimo Tránsito.
Kant, I. (2006). Idea de una historia universal con propósito cosmopolita. En I. Kant, Defensa de la ilustración (pp. 73-92). Barcelona: Alba.

Kneller, J. (2011). Nur ein Gedanke. Ein Kommentar zum Dritten und Vierten Satz von Kants Idee. En O. Höffe (Ed.), Immanue Kant. Schriften zur Geschichtsphilosophie (pp. 45-62). Berlín: Akademie Verlag.

Muchnik, P. (2009). Kant's Theory of Evil. An Essay on the Dangers of Self-Love and the Aprioricity of History. Maryland: Lexington Books.

Rousseau, J. J. (1990). Discurso sobre el origen y los fundamentos de la desigualdad entre los hombres. Madrid: Tecnos.

Schneewind, J. B. (2009). Good out of evil: Kant and the idea of unsocial sociability. En A. Oksenberg Rorty \& J. Schmidt (Eds.), Kant's Idea for a Universal History with a Cosmopolitan Aim. A Critical Guide (pp. 94-111). Cambridge: Cambridge University Press.

Wood, A. W. (1999). Kant's Ethical Thought. Cambridge: Cambridge University Press.

Wood, A. W. (2009). Kant's Fourth Proposition: the unsociable sociability of human nature. En A. Oksenberg Rorty \& J. Schmidt (Eds.), Kant's Idea for a Universal History with a Cosmopolitan Aim. A Critical Guide (pp. 112-128). Cambridge: Cambridge University Press.

Zotta, F. (2000). Immanuel Kant. Legitimität und Recht. Eine Kritik seiner Eigentumslehre, Staatslehre und seiner Geschichtsphilosophie. Múnich: Karl Alber. 


\section{Notas al final}

1 Como es la práctica común, las obras de Kant serán citadas conforme a la paginación de la edición de la Academia (Akademie Ausgabe). La forma de citar y referir será indicando el título original de la obra en alemán, la abreviatura alemana más común de la obra en cuestión -que indicaré la primera vez que haga referencia a una obra-, el volumen de la edición de la academia donde dicha obra está contenida, y la página correspondiente. La única excepción en esta forma de citación será, como es habitual en los estudios kantianos, la Kritik der reinen Vernunft $(\mathrm{KrV})$, la cual se citará con las siglas A y B correspondientes a la primera y segunda edición, y las páginas aludidas en cuestión. En los casos en los que no se consigna en la bibliografía final una traducción ya existente al castellano, la traducción de los pasajes de la obra en cuestión ha sido propia. Respecto de todas las traducciones, sin embargo, se cotejó siempre en paralelo el texto original alemán, haciendo en ocasiones ajustes menores a las mismas.

2 Idee zu einer allgemeinen Geschichte in weltbürgerlichen Absicht (IaG, Vol. AA VIII, p. 18 / Kant, 2006, p. 74). Considero que esta aseveración de Kant, no obstante, bien podría no ser otra cosa más que un recurso retórico para indicar que, difícilmente, en esta materia, hemos de llegar algún día a un conocimiento mucho más acabado, y que, más bien, sólo cabría esperar completar o corregir algunos aspectos de lo que aquí Kant presenta. El tipo de conocimiento que en este caso se ensaya -el cual busco explicar en la siguiente nota a pie con un mayor detalle- no podría dejar de ser, por su objeto propio, más que regulativo. De lo contrario, cabría conjeturar que estarían transgrediéndose ciertos límites trazados por el propio proyecto crítico. Las cuidadosas estipulaciones que Kant introduce más tardíamente en su obra para precisar en qué sentido puede hablarse de progreso dan cuenta de ello. Cfr. Der Streit der Fakultäten (SF, Vol. AA VII, pp. 79ss / Kant, 2003, pp. 151ss).

3 El juicio teleológico reflexionante alberga precisamente una conexión con la "idea" (Idee) en sentido kantiano -un término que queda recogido desde el título mismo del escrito que nos ocupa en este trabajo. Así pues, por ejemplo, leemos en la Kritik der Urteilskraft $(K U)$ : "Es suficiente con que se den objetos explicables única y exclusivamente conforme a leyes naturales que sólo podemos pensar bajo la idea (Idee) de fin como principio, objetos que, asimismo, sólo resulten internamente cognoscibles de esa manera según su forma interna. (...) (E)n la teleología se habla, ciertamente, de la naturaleza como si la finalidad en ella fuera intencional, pero al mismo tiempo ese propósito se atribuye a la naturaleza, esto es, a la materia, con lo cual (...) se quiere indicar que esta palabra sólo denota un principio de la facultad de juzgar reflexionante, no de la facultad de juzgar determinante (...)" (KU, Vol. AA V, p. 383 / Kant, 2003, pp. 362-363). Por supuesto, en este sentido, no debe pasarse por alto que para Kant las ideas son nociones que permiten asir o capturar algo más allá de la experiencia, con el fin de organizar de la mejor forma posible un conjunto o una totalidad, pero siempre en términos regulativos, no constitutivos ( $K r V$ A671/B699). Las ideas nos permiten contemplar el mundo, según la expresión kantiana, desde la perspectiva de un focus imaginarius ( $\mathrm{KrV}$ A 644/672).

4 Acerca del alma I, 12, 434a30-32 (Aristóteles 1978, p. 251); Partes de los animales I, 1, 641b12-26 (Aristóteles 2000, pp. 60-61); Marcha de los animales 704b11-17 (Aristóteles 2000, p. 266).

$5 \quad$ Este argumento, con algunas diferencias de tono, es empleado por Kant en otro importante contexto. Para distanciarse de los modelos éticos de corte eudaimonista, Kant niega en la Grundlegung der Metaphysik der Sitten (GMS) que sea la felicidad el fin más inmediato que la naturaleza ha deparado para los seres humanos (cfr. GMS, Vol. AA IV, pp. 394-396). De ser esto así, se conjetura ahí, ésta habría obrado más sabiamente en delegar esa tarea al instinto. A riesgo de suponer arbitrariedad o una equivocada asignación de sus medios y de sus recursos - cosa que no parece razonable hacer- hemos de suponer más bien que la naturaleza ha deparado otra tarea para nosotros, a saber, la de hacernos dignos de la felicidad mediante la buena conducta moral. Se puede ver con esto la importancia del apelo en diversos escenarios a la teleología, pues ésta ayuda a Kant a perfilar su proyecto de filosofía moral y a entender el entramado histórico humano. De ahí, por ende, que en este punto posturas que parecen restringir las consideraciones teleológicas a la narrativa histórica humana no sean del todo precisas, como por ejemplo la de Brandt (2010, p. 166).

6 Quien ha hecho un trabajo muy destacado de reconstrucción histórica de estos y algunos otros antecedentes es Schneewind (2009, pp. 94-111).

$7 \quad$ Un pasaje paralelo que, a mi entender, arroja una importante luz es uno contenido en la lección de la Vorlesung Anthropologie Friedländer. Presento mi traducción del mismo:

Hay pues en el hombre un principio de la sociedad y de la sociabilidad; y por otra parte hay un principio de la insociabilidad y de separación de la sociedad. Aquí colisionan los dos principios uno con otro; algo que no obstante está sabiamente dispuesto por el Creador. Primeramente, los seres humanos tienen el impulso de entrar en sociedad, pero, a fin de que la sociedad no permanezca agrupada en un solo lugar, los seres humanos tienen otro principio de la insociabilidad que los separa. Y así, de esta forma, la Tierra entera es poblada. Así pues, cuando la sociedad en un lugar es demasiado amplia, la gente se separa y va a otro lugar, p. ej. América. Cuando se multiplique la gente ahí también, se separará de nueva cuenta y habitará nuevas tierras. En último término, la tierra entera debe ser habitada. Cuando hay muchos Estados juntos éstos se unen y uno devora a los demás. Pero cuando uno se vuelve demasiado grande, éste se divide y los miembros buscan separarse. Éstas son la combinación y separación especiales del Creador a partir de las cuales surge la multiplicidad y a partir de la cuales después la completa perfección del género humano debe derivarse. Pues habrá de surgir un método de gobierno que habrá de mantenerse. (V-Anth/Friedländer, Vol. AA XXV, pp. 586-587).

A mi entender, los pasajes tienen énfasis distintos, pero concuerdan en puntos importantes: 1) hay distintas necesidades y apremios que llevan a los seres humanos unirse y separarse, 2) hay ciertas aptitudes o instituciones que se generan a partir de este proceso, 3 ) dicho proceso puede concebirse como teleológicamente guiado u orientado (en el caso de $I a G$ se habla, en 
términos más modestos, del plan de la naturaleza, mientras que en $V$-Anth/Friedländer se alude explícitamente a Dios como quien da la pauta de este desarrollo), y 4) la realización de la historia implica cierta compleción moral del género humano.

$8 \quad$ Esta posición es llamada por el propio Wood como una lectura antropológica del mal. El siguiente pasaje da cuenta de la misma:

Primero, la Religión confirma el contenido antropológico del mal, el cual se centra en los vicios de la competitividad humana. Pero, en segundo lugar, no obstante, la lectura antropológica de la doctrina del mal radical también implica que el mal tiene su origen en las comparaciones sociales y en los antagonismos. (...) Kant explícitamente atribuye la corrupción de la naturaleza humana a la condición social de los seres humanos, y de modo más específico, a la inclinación de un amor propio en términos comparativos que caracteriza a las personas cuando viven en proximidad unas con las otras. Considerados abstractamente, los deseos naturales de los seres humanos son moderados, y están dispuestos para que uno halle contento. Lo que interfiere con este contento es el miedo del ser humano de ser visto por los otros como carente de valor. (Wood, 1999, p. 288).

Traduciré directamente al castellano las citas de comentadores de lengua inglesa y alemana. En otro trabajo reciente, Wood (2009) ha equiparado nuevamente el mal radical con la sociable insociabilidad. Una obra donde se hace un desarrollo interpretativo que guarda grandes afinidades con el de Wood es la de Anderson-Gold (2001).

9 Ver Allison (2001, pp. 594-616).

$10 \quad$ Die Religion innerhalb der Grenzen der bloßen Vernunft (RGV).

11 Conviene traer a colación un pasaje rousseauniano para ver los antecedentes de la concepción kantiana del mal incoado en la convivencia social. Se trata de una nota del Segundo discurso:

No se pueden confundir el amor propio (amour propre) y el amor a sí mismo (amour de soi), dos pasiones muy diferentes por su naturaleza y por sus efectos. El amor a sí mismo es un sentimiento natural que lleva a todo animal a preocuparse por su conservación y que, dirigido en el hombre por la razón y modificado por la piedad, da por resultado la humanidad y la virtud. El amor propio es tan sólo un sentimiento relativo, artificial y nacido dentro de la sociedad, que lleva a cada individuo a ocuparse más de sí que de cualquier otro, que inspira a los hombres todos los males que se perpetran mutuamente y que es la verdadera fuente del honor. (Rousseau, 1990, p. 235).

Es de recordar que, para Rousseau, el amor propio no existe en el así llamado estado de naturaleza. Por estas afinidades, podría decirse que la lectura que emprende Wood de Kant cabría ser caracterizada como rousseauniana.

12 Es cierto que el Religionsschrift es un escrito más tardío que IaG: el primero fue publicado en 1792 mientras que el segundo apareció en 1784. No obstante, en términos sistemáticos, no veo dificultad en trazar el puente entre ambos escritos, en los términos en lo que lo planteo, toda vez que en $I a G$ no encuentro elemento alguno que sugiera que de lo que se está dando cuenta ahí es del origen del mal moral. Por lo mismo, cabría pensar que esta tarea quedó pendiente y que sólo fue consumada hasta el Religionsschrift.

13 Mi exposición de lo que Kant afirma sobre el mal radical pone de relieve rasgos que, me parece, son bastante aceptados e incontrovertidos. Pero para una discusión a este respecto bastante más detallada, véase por ejemplo el excelente estudio de Muchnik (2009).

14 Por lo demás, posiblemente se incurre en un error al pensar en toda forma de insociabilidad como una forma o manifestación de un mal moral. Podemos pensar sin muchas dificultades en conductas antisociales que no necesariamente emanan de un sentimiento de misantropía. Uno podría actuar de forma antisocial por temor a sufrir algún tipo violencia o por no considerarse seguro en algún tipo de contexto. En el pasaje de $I a G$ de la cuarta proposición, se aprecia además que este inicial alejamiento o aislamiento se da por una resistencia de los demás a los propios anhelos o deseos. Por supuesto, esta insociabilidad "neutra" moralmente podría mutar fácilmente después -como de hecho lo hace- en todo lo que Kant concibe como vicios propios de la cultura.

15 Hay que decir, a su vez, que sin esta dimensión individual del mal no cabría pensar o concebir un proceso de perfeccionamiento moral al que, a pesar de las circunstancias, estaríamos llamados. Y es de recordar también en la propuesta kantiana la importancia que cobran los deberes hacia uno mismo (Metaphysik der Sitten [MS], Vol. AA VI, pp. 421ss). Es posible concebir que, aun si uno estuviese retirado de un determinado contexto comunitario, seguiríamos estando obligados a observar dichos deberes, y la razón detrás de ello es que podría darse un mal moral que no fuese social.

16 Kant menciona expresamente que los estoicos erraron al concebir que, el enemigo con el cual uno tenía que vérselas en materia moral, era la sensibilidad o la naturaleza. El filósofo alemán advierte que éste es un error porque la naturaleza en sí no es como tal imputable o reprochable. Antes bien, habría que reconocer ese enemigo en nosotros mismos y en nuestros usos desviados de la libertad ( $R G V$, Vol. AA VI, pp. 57ss / Kant, 2001, pp. 77ss; ver también $M S$, Vol. AA VI, p. 380). En este sentido, me parece, Wood se vuelve con su interpretación objeto de este reproche kantiano, pues termina por hacer del mal, de algún modo u otro, algo constitutivo de la naturaleza humana.

17 Y más aún, aunque esto no lo diga Kant expresamente con esta terminología, uno podría pensar que el mal también es susceptible de manifestarse en ciertas formas, algunas más decadentes que otras, de sociabilidad. Piénsese, por ejemplo, en la convivencia que puede surgir entre algunos infringiendo los deberes de respeto e incidiendo en conductas como la maledicencia (Afterreden) y la burla (Verhöhnung) en contextos de camaradería (MS, Vol. AA VI, pp. 466-469). E incluso, en términos más especulativos, uno podría decir o pensar incluso que obrar conforme al deber (pflichtgemäß), únicamente en virtud de no sufrir ciertas repercusiones como caer en descrédito ante los demás, según el famoso ejemplo de GMS del comerciante, podría ser con- 
siderado como una forma impropia o dudosa de ser social (GMS, Vol. AA IV, p. 397).

18 Esto se alcanza a apreciar también, por ejemplo, en la discusión en la $K U$ sobre el summum bonum, mismo que es justificado ahí en clave teleológica. Parte de la necesidad práctica de creer en el mismo estriba en que, de no hacer tal cosa, fácilmente podríamos claudicar y desesperar en el afán de llevar una buena conducta, pues veríamos que la naturaleza sólo coincide, en el mejor de los casos, accidentalmente con nuestros esfuerzos morales y que, según la dura caracterización kantiana, sólo tendríamos ante nuestros ojos el prospecto de perecer sin más como cualquier otra creatura sobre la tierra. Véase ( $K U$, Vol. V AA, pp. 447-454 / Kant, 2003, pp. 437-443).

19 Otra consideración sistemática interesante que Höffe presenta es la siguiente: no es inverosímil pensar que un gobernante medianamente bueno genere instituciones que a la postre puedan considerarse como buenas, y viceversa, no es imposible pensar, por ejemplo, que una ciudadanía medianamente buena pueda presentar ciertas exigencias que a la larga terminen por disuadir a los gobernantes de perseguir la guerra, y con ello sentar las bases para la instauración de un orden republicano (Höffe, 2006, p. 175).

20 Por otra parte, esta crítica resulta todavía más desconcertante proviniendo de Arendt, siendo que ella, con gran lucidez, identifica cómo las acciones se entrelazan unas con otras, y cómo las mismas pueden tener efectos no previstos del todo por los individuos, Esto, a mi parecer, no varía esencialmente de lo que Kant estaría diciendo. La diferencia estribaría en qué Kant, desde las coordenadas propias del criticismo trascendental, estaría brindando una clave o hilo heurístico para interpretar esos mismos procesos. Véase sobre esto: Arendt (1998, pp. 181ss). Por lo demás, es de resaltar que esta misma opinión sobre la instrumentalización de los seres humanos a través de la historia ha vuelto a aparecer en algunos comentadores recientes. Véase Brandt (2010, p. 166) y Zotta (2000, pp. 179ss). Considero, sin embargo, que el núcleo de lo que dicen no es esencialmente distinto de lo apuntado en su momento por Arendt.

21 Además de todo, la clave de lectura de Kneller no tiene plena consistencia teórica con el resto de la $K U$, obra en la que su análisis se basa. En efecto, en la tercera Crítica, Kant hace desarrollos muy semejantes a los de IaG para subrayar el fin último de la naturaleza como aquella cultura de la disciplina (Cultur der Zucht) que el ser humano tendría que desarrollar para librarse de sus apetitos más rudos y poder poner fin, mediante la federación republicana de naciones, a las hostilidades que acongojan a su género. Cfr. ( $K U$, Vol. AAV, pp. 432-433). Por supuesto, esto no quiere decir que el así llamado proceso de la sociable sociabilidad que Kneller destaca no exista en lo absoluto en Kant. Claramente, sí existe esa vinculación entre estética y moral en lo que sería la sociabilidad del ser humano. Pero su análisis peca por no explicar cómo esto estaría relacionado con ese otro proceso colectivo de la insociable sociabilidad al cual Kant vuelve a recurrir en $K U$, y cómo o bajo qué condiciones uno podría suplantar o sustituir al otro.

Véase la nota anterior.

(MS, Vol. AA VI, pp. 405ss). Un excelente análisis a este respecto se encuentra en De Haro (2015, pp. 176ss). 